\title{
Dispersion energy analysis of Rayleigh and Love waves using finite-difference modeling
}

\author{
Binbin $\mathrm{Mi}^{1}$, Jianghai Xia ${ }^{1,2}$, Chao Shen ${ }^{1}$, and Limin Wang ${ }^{1,2}$ \\ ${ }^{1}$ Subsurface Imaging and Sensing Laboratory, Institute of Geophysics and Geomatics, China University of Geosciences (Wuhan) \\ ${ }^{2}$ Hubei Subsurface Multi-scale Imaging Key Laboratory, Institute of Geophysics and Geomatics, China University of Geosciences (Wuhan)
}

\begin{abstract}
Surface-wave analysis methods have been effectively and widely used to determine shear (S) wave velocity. To separate and identify different dispersive modes of surface waves accurately is one of key steps using surface-wave methods. We analyze the dispersion energy of Rayleigh and Love waves based on staggered-grid finite-difference modeling in 2D isotropic elastic media with horizontally homogeneous layered models. Results demonstrate that when there is a low velocity layer (LVL) in an earth model, LVL-guided waves will generate and possess energy on dispersive images, which can interfere with the dispersion energy of Rayleigh or Love waves. The dispersive energy looks like "jumping" from the fundamental mode to higher modes on dispersive images because of dispersion curves (whatever the fundamental or higher modes) of LVL-guided waves being lack of energy in a high-frequency range. If the $S$ wave velocity of the LVL is higher than the surface layer, the energy of LVL-guided waves only contaminates higher mode energy of surface waves and there is no interlacement with the fundamental mode of surface waves. While if the S-wave velocity of the LVL is lower than the surface layer, the energy of LVLguided waves may interlace with the fundamental mode of surface waves. This may cause misidentification for the fundamental dispersion curve of surface waves and produce significant errors in the inversion.
\end{abstract}

Keywords: Rayleigh and Love waves, dispersion, LVL

\section{INTRODUCTION}

Surface waves, Rayleigh and Love waves, travel along a "free" surface, such as the earth-air or the earth-water interface and are usually characterized by relatively low velocity, low frequency, and high amplitude. Today, surface-wave analysis is widely adopted for building shear (S) wave velocity models at a multiple scales-global seismology, exploration geophysics, near-surface geophysics. All of these applications share the same principles: they use the dispersive characteristic of surface waves to infer the properties of the medium by identifying the model parameters. In near-surface applications, the multichannel analysis of surface wave (MASW) and multichannel analysis of Love wave (MALW) methods have been given increasingly more attention and widely used to determine near-surface S-wave velocities during the past two decades ${ }^{[5]}$.

In the real world applications, the complexity of energy distribution on a dispersion image is exacerbated due to the complicated subsurface earth model. Previous studies about a low velocity layer (LVL) among a layered earth model found that dispersive energy of such model "jumps" from the fundamental mode to higher modes and may not return to the fundamental mode at higher frequencies, which brings a pitfall of mode-misidentification and produces incorrect inversion results ${ }^{[7]}$. Safani et al. (2006) also showed that it is difficult to identify Love-wave mode when a low-velocity layer (LVL) is bounded by equally stiff layers ${ }^{[4]}$.

Surface-wave wavefield modeling on the basis of elastodynamic equations can present the propagation and dispersion characteristics of surface waves accurately. We propose to analyze the dispersion energy of Rayleigh and Love waves based on staggered-grid finite-difference modeling in 2D isotropic elastic media with horizontally homogeneous layered model ${ }^{[3]}$. The dispersion images in the $\mathrm{f}-\mathrm{v}$ domain are generated from the synthetic shot gathers to present energy distribution of Rayleigh and Love waves. The resolution limitation of the dispersion image constrains the recognition of peak values of energy concentration. Previous research showed that the resolution of dispersion images generated by the high-resolution linear Radon transformation (LRT) is $50 \%$ higher than those of the other methods ${ }^{[2]}$. Therefore we use the high-resolution LRT technique to image dispersive energy for all the synthetic data of the investigated models throughout this paper.

\section{When Does The DisPersion ENERGY "JUMP"}

In order to study the propagation and dispersion characteristics of Rayleigh and Love waves tentatively, we first designed three three-layer models (Table 1) and performed dispersive analysis on the modeled Rayleigh and Love waves. In the finite-difference modeling of Rayleigh and Love waves, the source is a $20 \mathrm{~Hz}$ (peak frequency) Ricker wavelet with $60 \mathrm{~ms}$ delay, located at the free surface. Seismic responses are recorded on the free surface with a 60-channel receiver array. The nearest offset is $30 \mathrm{~m}$, with a subsequent 1 $\mathrm{m}$ receiver interval.

The first model in Table 1 represents a normal layered model with $\mathrm{P}$ and $\mathrm{S}$-wave velocities increasing with depth. The dispersive images of Rayleigh and Love waves are shown in Figs. 1a and b. Rayleigh and Love-wave energy dominates the two images, respectively. Dispersive energy concentrates for each mode distinctly and continuously, and it does not disappear at higher frequencies. Comparing Figures 1a and b, it is worth mentioning that Love waves have a wider frequency band than Rayleigh waves with the finite-difference modeling method. 
The second model in Table 1 contains a high velocity layer (HVL). Fig.1c and $\mathrm{d}$ show the corresponding dispersive images of Rayleigh and Love waves. Rayleigh and Love-wave energy dominates the two images, respectively. Dispersive energy concentrates for each mode distinctly and continuously, and it does not disappear at higher frequencies.

The third model in Table 1 contains a low velocity layer (LVL). Fig. 1e and f show the corresponding dispersive images of Rayleigh and Love waves. However, there is almost no energy where the phase velocities are smaller than $300 \mathrm{~m} / \mathrm{s}$ both in Figures 1e and $\mathrm{f}$. The dispersive energy "jumps" from the fundamental mode to higher modes, and the dispersion curves (whatever the fundamental or higher modes) lack energy in a high-frequency range. The energy trends from low phase velocities to high phase velocities with the increasing frequencies.

Dispersion analysis of three-layer models above reveals some critical characteristics of Rayleigh and Love-wave propagation in a LVL model. Results demonstrate that the dispersion energy "jumps" when there is a LVL in the earth model. Under this circumstance, another problem arises. It is known that the existence of Love wave was first predicted for a homogeneous layer overlying a homogeneous half-space with an S-wave velocity greater than that of the layer ${ }^{[1]}$. For Model 3 with a LVL, the S-wave velocity of second layer is lower than that of top layer, so Love waves will not generate between the two layers. Then why is still there the dispersion energy in Figure 1f and what is it?

\section{Why DOES THE DisPersion ENERGY "JUMP"}

Guided waves are trapped in a waveguide by total reflections or bending of rays at the top and bottom boundaries [1]. If we consider the Earth's surface as the top of a waveguide, surface waves, such as Rayleigh, Love, and their higher modes, are guided waves. The waves associated with a low-velocity channel in the crust or mantle may be interpreted as normal modes with concentration of energy in the channel ${ }^{[1]}$. In nearsurface applications, if there is a LVL in the earth model, one kind of guided waves will generate and propagate in that layer where most of the energy is trapped. We call this kind of guided waves as LVL-guided waves, distinguished from other guided waves like surface waves. LVL-guided waves are also dispersive because longer wavelengths can penetrate out of the LVL for a given mode, generally exhibit greater phase velocities, and are more sensitive to the elastic properties of the top and bottom layers. Conversely, shorter wavelengths with lower phase velocities are trapped in the LVL.

Shot gathers on the free surface can record all the seismic waves that spread to the surface and dispersive images generated from the shot gather contain full wavefield information. Therefore, dispersion energy contains the information of all kinds of guided waves. If there is a LVL in the earth model, LVL-guided waves will generate and possess energy on dispersive images, which can interfere with the dispersion energy of Rayleigh or Love waves and cause mode misidentification. That is why the dispersion curves are more complicated when there is a LVL in the earth model. Moreover, shorter wavelengths of LVL-guided waves may not penetrate the free surface, then the dispersion curves (whatever the fundamental or higher modes) lack energy in a highfrequency range. As a consequence, the dispersive energy looks like "jumping" from the fundamental mode to higher modes.

Different from Rayleigh waves, Love-wave generation requires the existence of a low velocity surface layer in a multilayered earth model. If the S-wave velocity of second layer is lower than that of top layer, Love waves will not generate between the two layers. While if the S-wave velocity of second layer is lower than that of top and bottom layers, LVL-guided waves will generate in that low velocity layer. That is why there is still dispersion energy in Fig. If for the third three-layer model in Table 1 with a LVL.

In order to validate the explanation above, we designed a six-layer model with two LVLs (Table 2) and performed dispersive analysis on the modeled Rayleigh and "Love" waves. In the finite-difference modeling, the source is a $20 \mathrm{~Hz}$ (peak frequency) Ricker wavelet with $60 \mathrm{~ms}$ delay, located at the free surface. Seismic responses are recorded on the free surface with a 60-channel receiver array. The nearest offset is $30 \mathrm{~m}$, with a subsequent $1 \mathrm{~m}$ receiver interval.

The six-layer model in Table 2 contains two LVLs (the second and fourth layers). As the analysis above, two series of LVL-guided waves will generate in the second and fourth layers, respectively. Certainly, Rayleigh wave will also produce, but there should not be Love wave because the Swave velocity of the top layer is higher than that of second layer. Fig.2a and $\mathrm{b}$ show the corresponding dispersive images of Rayleigh and "Love" waves. Fig.2a shows energy distribution of Rayleigh waves and guided waves generated in the two LVLs based on P-SV wave system. $\mathrm{R}_{1}$, with the asymptote at the high frequency approaching about 0.9 times S-wave velocity of top layer, represents the fundamental energy of Rayleigh waves. However, the higher mode energy of Rayleigh waves and the energy of LVL-guided waves are complicated and difficult to identify. By contrast, the energy distribution in Figure $2 b$ is simpler, which is the guided-wave energy generated in the two LVLs based on SH wave system. $\mathrm{G}_{2-1}, \mathrm{G}_{2-2}, \mathrm{G}_{2-3}$ and $\mathrm{G}_{2-4}$, lacking energy in a high-frequency range and not approaching $\mathrm{S}$-wave velocity of top layer, represent the energy of the fundamental and the first, second, third higher modes of the LVL-guided waves generated in the second LVL, respectively; $\mathrm{G}_{4-1}, \mathrm{G}_{4-2}, \mathrm{G}_{4-3}$ and $\mathrm{G}_{4-4}$, having energy only in a greater wavelength range, represent the energy of the fundamental and the first, second, third higher modes of the LVL-guided waves generated in the fourth LVL, respectively. There is no Love wave energy in Fig.2b because there is no asymptote of energy at the high frequency approaching S-wave velocity of top layer.

The asymptotes of LVL-guided wave dispersion curves at the high frequency should approach S-wave velocity of the LVL. However, the shot gather is recorded on the free surface and the LVL has a distance to the free surface in the earth 
model. Thus, shorter wavelengths of LVL-guided waves may not penetrate to the free surface, then the dispersion curves (whatever the fundamental or higher modes) lack energy in a high-frequency range. This is different from the properties of Rayleigh and Love waves, whose shorter wavelengths travel around the free surface and dispersion curves still have energy in a high-frequency range. This principle can be used to identify whether the energy on the dispersive image is surface wave or LVL-guided wave.

TABLE I.

PARAMETERS OF THREE-LAYER MODELS

\begin{tabular}{c|c|c|c|c|c|c|c|c|c}
\hline \multirow{2}{*}{ Layer number } & \multicolumn{3}{|c|}{ Vp $(\boldsymbol{m} / \mathbf{s})$} & \multicolumn{3}{c|}{ Ds $(\boldsymbol{m} / \mathbf{s})$} & \multirow{2}{*}{$h(\boldsymbol{m})$} \\
\cline { 2 - 10 } & Model 1 & Model 2 & Model 3 & Model 1 & Model 2 & Model 3 & \\
\hline 1 & 800 & 800 & 1200 & 200 & 200 & 400 & 2.0 \\
\hline 2 & 1200 & 1600 & 800 & 400 & 600 & 200 & 2.0 \\
\hline 3 & 1600 & 1200 & 1600 & 600 & 400 & 600 & 2.0 \\
\hline
\end{tabular}

TABLE II. PARAMETERS OF A SIX-LAYER MODEL WITH TWO LVLS

\begin{tabular}{c|c|c|c|c}
\hline Layer number & $\mathrm{Vp}(\boldsymbol{m} / \mathbf{s})$ & $\mathrm{Vs}(\boldsymbol{m} / \mathbf{s})$ & Density $(\mathbf{g} / \mathbf{c m} \mathbf{3})$ & $h(\boldsymbol{m})$ \\
\hline 1 & 650 & 194 & 1.82 & 2 \\
\hline 2 & 550 & 125 & 1.86 & 2 \\
\hline 3 & 1400 & 367 & 1.91 & 3 \\
\hline 4 & 600 & 137 & 1.96 & 3 \\
\hline 5 & 2150 & 603 & 2.02 & 4 \\
\hline 6 & 2800 & 740 & 2.09 & Infinite \\
\hline
\end{tabular}

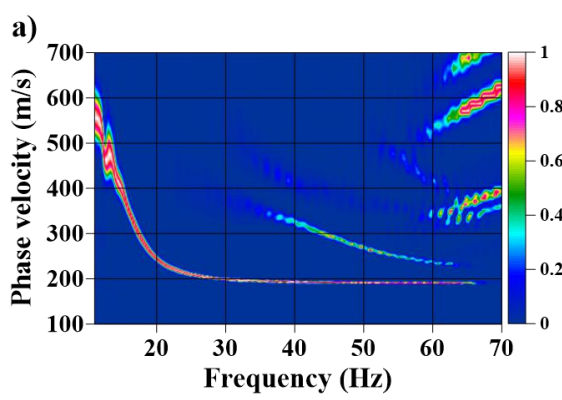

b)
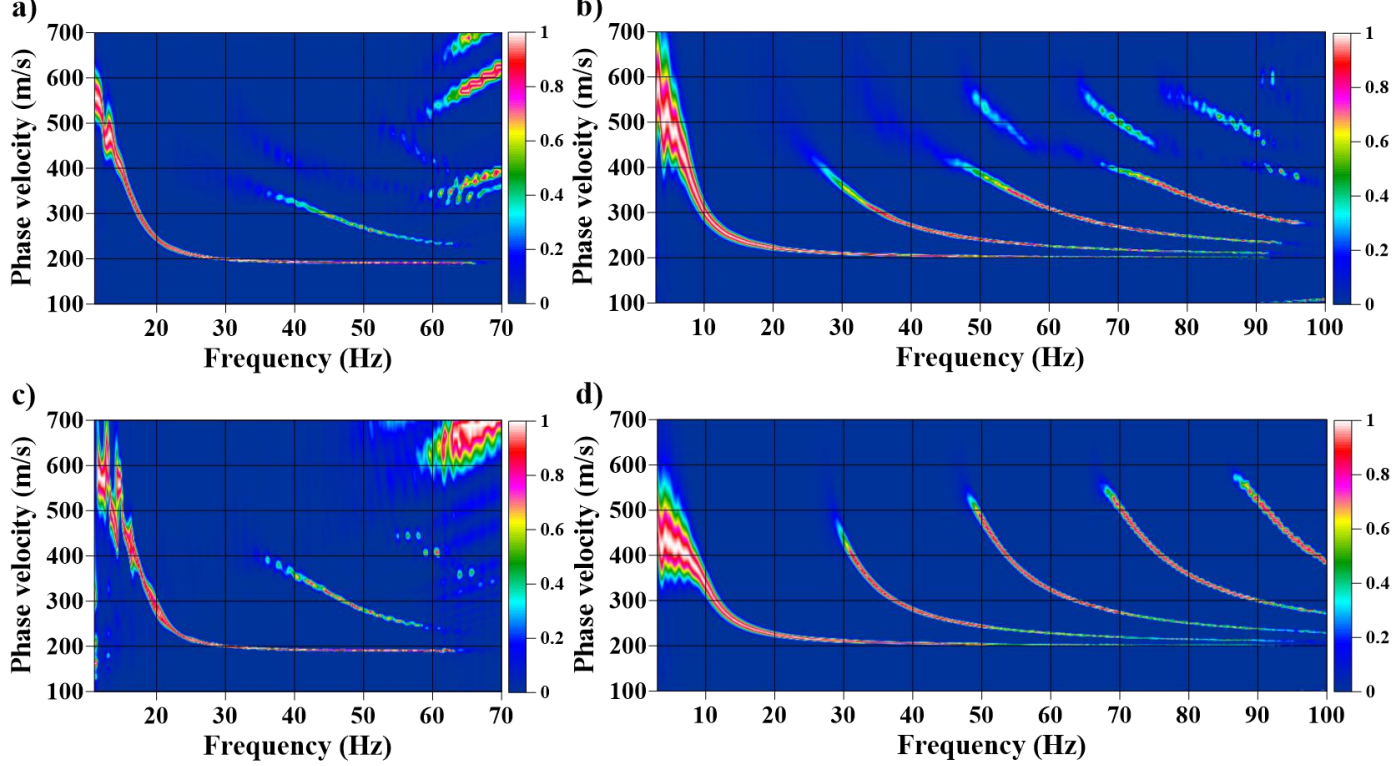

d)

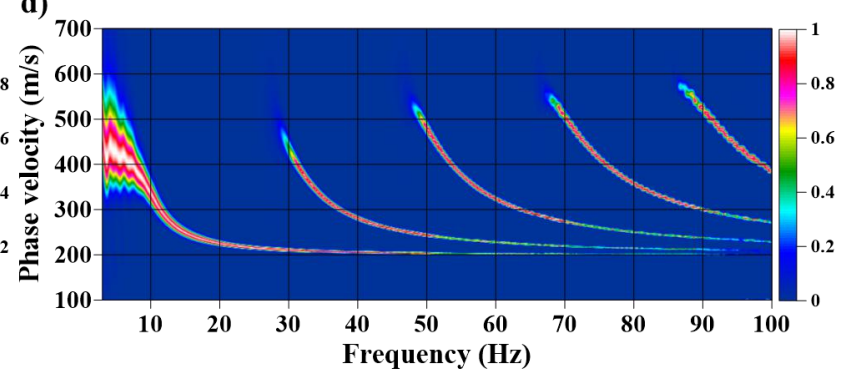

e)

f)
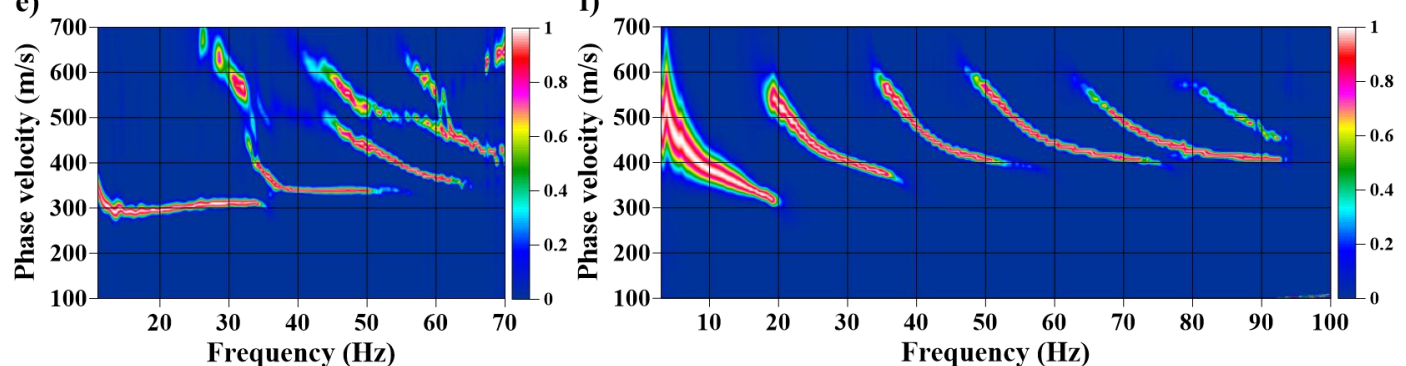

Fig. 1. Dispersion images in the $\mathrm{f}-\mathrm{v}$ domain for the three-layer models in Table 1. Rayleigh-wave energy distribution is showed in (a), (c), and (e) for Model 1, Model 2, and Model 3, respectively. Love-wave energy distribution is showed in (b), (d), and (f) for Model 1, Model 2, and Model 3, respectively. 
a)

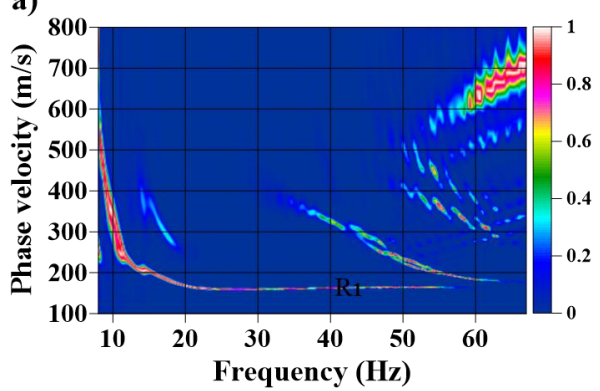

b)

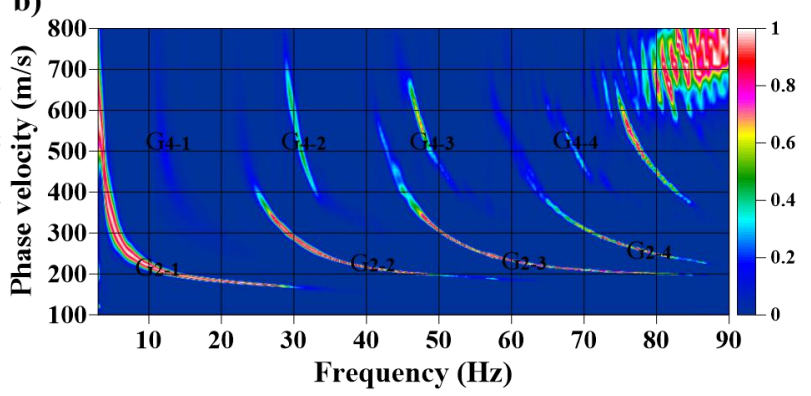

Fig. 2. Dispersion images in the $\mathrm{f}-\mathrm{v}$ domain for the six-layer model with two LVLs in Table 2. (a) shows energy distribution of Rayleigh waves and guided waves generated in the two LVLs based on P-SV wave system. R1 represents the fundamental energy of Rayleigh waves; the higher mode energy of Rayleigh waves and the energy of LVL-guided waves is difficult to identify. (b) shows energy distribution of the guided waves generated in the two LVLs based on SH wave system. G2-1, G2-2, G2-3 and G2-4 represent the energy of the fundamental and the first, second, third higher modes of the LVL-guided waves generated in the second LVL, respectively; G4-1, G4-2, G4-3 and G4-4 represent the energy of the fundamental and the first, second, third higher modes of the LVL-guided waves generated in the fourth LVL, respectively. There is no Love wave energy in (b).

\section{CONCLUSIONS}

We have analyzed the dispersion energy of Rayleigh and Love waves based on staggered-grid finite-difference modeling in 2D isotropic elastic media with horizontally homogeneous layered models. Results demonstrate that when there is a LVL in the earth model, LVL-guided waves will generate and possess energy on dispersive images, which can interfere the dispersion energy of Rayleigh or Love waves. The dispersive energy looks like "jumping" from the fundamental mode to higher modes on dispersive images because of dispersion curves (whatever the fundamental or higher modes) of LVL-guided waves lack energy in a highfrequency range. If the S-wave velocity of the LVL is lower than the surface layer, the energy of LVL-guided waves may interlace with the fundamental mode of surface waves. This may cause great misidentification for the fundamental dispersion curve of surface waves and produce significant errors in the inversion.

\section{ACKNOWLEDGMENT}

This research is supported by the National Natural Science Foundation of China (NSFC, Grant No. 41274142) and the National Nonprofit Institute Research Grant of Institute for Geophysical and Geochemical Exploration, Chinese Academy of Geological Sciences (Grant No. WHS201307).

\section{REFERENCES}

[1] Aki, K. and Richards, P.G., Quantitative Seismology (2nd ed.), University Science Books, 2002.

[2] Luo, Y., Xia, J., Miller, R.D., Xu, Y., Liu, J., and Liu, Q., 2008. Rayleigh-wave dispersive energy imaging by high-resolution linear Radon transform. Pure and Applied Geophysics, 165(5), 903-922.

[3] Luo, Y., Xia, J., Xu, Y., Zeng, C., Liu, J., 2010. Finite-difference modeling and dispersion analysis of high-frequency Love waves for near-surface applications. Pure Appl. Geophys.167 (12), 1525-1536.

[4] Safani, J., O’Neill, A. and Matsuoka, T. (2006), Full SH-wavefield modelling and multiple-mode Love wave inversion , Explor. Geophys. 37, 307-321.

[5] Xia, J., 2014. Estimation of near-surface shear-wave velocities and quality factors using multichannel analysis of surface-wave methods. Journal of Applied Geophysics, 103, 140-151.

[6] Xu, Y., Xia, J., Miller, R.D., 2007. Numerical investigation of implementation of air-earth boundary by acoustic-elastic boundary approach. Geophysics, 72(5), SM147-SM153.

[7] Zhang, S. X., and L. S. Chan, 2003, Possible effects of misidentified mode number on Rayleigh wave inversion: Journal of Applied Geophysics, 53, 17-29. 